\title{
A moving object baring angle measurement using orthogonal circle polarized radio beacon signals
}

\author{
V L Gulko ${ }^{1}$, A A Mescheryakov ${ }^{2}$ \\ ${ }^{1}$ Candidate of Engineering Sciences, Associate Professor, Researcher of the \\ Institute of Radio-engineering systems, TUSUR, Tomsk, Russia \\ ${ }^{2}$ Candidate of Engineering Sciences, Associate Professor, Lead researcher of the \\ Institute of Radio-engineering systems, TUSUR, Tomsk, Russia \\ E-mail: msch@rts.tusur.ru

\begin{abstract}
There is investigated the opportunity to find the moving object bearing angle using radio beacon signals polarized within the left and right circles. Signals are illuminated simultaneously from two space diversed in the horizontal plane points. Amplitude-phase processing of the vector signals received onboard the moving object and treated in the linear and circular polarized bases is utilized in order to find the bearing angle.
\end{abstract}

\section{Introduction}

In the practical navigation in order to find the moving object (MO) bearing angle there are used amplitude, frequency and time characteristics of the radio bacon signals [1]. The vector nature of the signals in the radio bacon systems (RBS) practically is not taken into consideration [2]. In [3] there was suggested a polarization method of the MO bearing angle measurement. The idea of the method is following. A radio bacon simultaneously illuminates signals with the vertical and horizontal polarization from two points horizontally separated by the distance $d$. Amplitudes, initial phases and the wave length of the illuminated signals are the same. The signals are received onboard the MO in the linear polarization basis (LPB). The phase difference of the signals $\Delta \varphi$ is measured after the polarization separation. The bearing angle $\alpha$ is the angle between the normal at the $d$ half distance and the direction to the $\mathrm{MO}[3]$ :

$$
\alpha= \pm \arcsin \left\{\frac{\lambda}{2 \pi d} \cdot \Delta \varphi\right\} \pm n \pi,
$$

where $n=0,1,2 \ldots$

$\lambda$ - the wave length of the illuminated orthogonal polarized waves.

In the paper there is investigated the opportunity to use the orthogonal polarized signals within the left and right circles to measure the $\mathrm{MO}$ bearing angle.

The choice of the polarization basis of the illuminated and received vector signals depends on peculiarities of the technical problem. 


\section{The method of the problem solution and the main relations}

Let's see the case when the bacon illuminates simultaneously signals with circle polarization equal amplitudes and initial phases and the same frequencies. The resulting vector signals are received onboard the MO in LPB.

The resulting wave in the direction $\alpha$ can be written as follows [4]:

$$
\overrightarrow{\dot{E}}_{p}=\frac{1}{2}\left\{\left[\begin{array}{c}
1 \\
-j
\end{array}\right]+\left[\begin{array}{l}
1 \\
j
\end{array}\right] \cdot e^{j \Delta \varphi}\right\},
$$

where $\Delta \varphi=\frac{2 \pi d}{\lambda} \sin \alpha$ - the phase difference between orthogonal left and right polarized waves at the point of the reception onboard the MO.

After dividing the phase shift $\Delta \varphi$ equally between the two waves the resulting wave is [4]:

$$
\vec{E}_{p}=\left[\begin{array}{c}
\cos \frac{\Delta \varphi}{2} \\
-\sin \frac{\Delta \varphi}{2}
\end{array}\right] .
$$

The equation (2) shows that the resulting wave is always polarized linearly and its orientation on the polarization plate depends on the phase shift $\Delta \varphi$. Its intensity does not depend on the direction of illumination as the waves are orthogonal.

The resulting wave is received onboard the MO with the antenna and UHF tract with linear polarization divider (LPD). Let's use the known formalism of Jones's vectors and matrixes [5] treatment to describe the interaction of the resulting wave (2) and the elements of LPD. In such a case $\vec{E}_{X}$ and $\vec{E}_{Y}$ at the LPD output can be found as:

$$
\begin{aligned}
& \vec{E}_{X}=\left[\Pi_{1}\right] \cdot \vec{E}_{P}, \\
& \vec{E}_{Y}=\left[\Pi_{2}\right] \cdot \vec{E}_{P},
\end{aligned}
$$

where $\left[\Pi_{1}\right]=\left[\begin{array}{ll}1 & 0 \\ 0 & 0\end{array}\right]$ - is the Jones's operator of the LPD first shoulder with its own horizontal polarization;

$$
\left[\Pi_{2}\right]=\left[\begin{array}{ll}
0 & 0 \\
0 & 1
\end{array}\right] \text { - is the Jones's operator of the LPD second shoulder with its own vertical }
$$
polarization.

After calculation (3) and (4) the orthogonal linearly polarized components $\vec{E}_{X}$ and $\vec{E}_{Y}$ at the LPD output are:

$$
\vec{E}_{X}=\left[\begin{array}{c}
\cos \frac{\Delta \varphi}{2} \\
0
\end{array}\right]
$$




$$
\vec{E}_{Y}=\left[\begin{array}{c}
0 \\
-\sin \frac{\Delta \varphi}{2}
\end{array}\right]
$$

Formulas (5) and (6) allow to calculate phases $\varphi_{X}$ and $\varphi_{Y}$ as well as the components $\vec{E}_{X}$ and $\vec{E}_{Y}$ ratio $A_{Y} / A_{X}$ at the output of the two-canal receiver with the linear amplitude characteristic and the linear detector:

$$
\begin{gathered}
\varphi_{X}=\varphi_{Y}=0^{\circ}, \\
\frac{A_{Y}}{A_{X}}=\left|\frac{\sin \Delta \varphi / 2}{\cos \Delta \varphi / 2}\right|=\left|\operatorname{tg} \frac{\Delta \varphi}{2}\right| .
\end{gathered}
$$

Then

$$
\Delta \varphi= \pm 2 \operatorname{arctg} \frac{A_{Y}}{A_{X}} \pm 2 \pi n
$$

where $n=0,1,2, \ldots$.

After putting (7) in to (1) the MO bearing angle $\alpha$ is

$$
\alpha= \pm \arcsin \left[\frac{\lambda}{\pi d}\left( \pm \operatorname{arctg} \frac{A_{Y}}{A_{X}} \pm \pi n\right)\right] .
$$

In case of the two-canal receiver with the logarithmic amplitude characteristic and linear detector (8) becomes

$$
\alpha= \pm \arcsin \left[\frac{\lambda}{\pi d}\left( \pm \operatorname{arctg} 10^{\lg \frac{A_{Y}}{A_{X}}} \pm \pi n\right)\right] .
$$

Let's investigate the case when the resulting wave (2) is received onboard the MO in the circle polarization basis $(\mathrm{CPB})$.

The wave (2) can be presented by two orthogonal polarized left $\dot{E}_{L}$ and right $\dot{E}_{R}$ vectors.

Let's find amplitudes $A_{L}$ and $A_{R}$, phases $\Psi_{L}$ and $\Psi_{R}$ of the components $\dot{E}_{L}$ and $\dot{E}_{R}$ and then find their dependence on the MO bearing angle $\alpha$.

The Jones vector $\vec{E}_{P}$ (2) can be found onboard the MO by the transformation (in the circle sinphase polarization basis when the time dependence is neglected):

$$
\overrightarrow{\dot{E}}_{L R}=\left[\begin{array}{c}
\dot{E}_{L} \\
\dot{E}_{R}
\end{array}\right]=[\theta] \cdot \vec{E}_{P}
$$

where $[\theta]=\frac{1}{\sqrt{2}}\left[\begin{array}{cc}1 & j \\ 1 & -j\end{array}\right]$ - is the operator of the transition from LPB to CPB [5].

After calculation (9) there are received orthogonal polarized left $\dot{E}_{L}$ and right $\dot{E}_{R}$ vectors at the input of the two-canal receiver: 


$$
\begin{aligned}
& \dot{E}_{L}=\frac{1}{\sqrt{2}}\left[\cos \frac{\Delta \varphi}{2}-j \sin \frac{\Delta \varphi}{2}\right], \\
& \dot{E}_{R}=\frac{1}{\sqrt{2}}\left[\cos \frac{\Delta \varphi}{2}+j \sin \frac{\Delta \varphi}{2}\right] .
\end{aligned}
$$

Amplitudes $A_{L}, A_{R}$ and phases $\Psi_{L}, \Psi_{R}$ of the signals (10) and (11) are:

$$
\begin{array}{ll}
A_{L}=\frac{1}{\sqrt{2}} ; & \Psi_{L}=-\operatorname{arctg} \frac{\sin \Delta \varphi / 2}{\cos \Delta \varphi / 2}, \\
A_{R}=\frac{1}{\sqrt{2}} ; & \Psi_{R}=\operatorname{arctg} \frac{\sin \Delta \varphi / 2}{\cos \Delta \varphi / 2} .
\end{array}
$$

The phase difference $\Delta \Psi_{R L}$ after transformation looks like

$$
\Delta \Psi_{R L}=\Psi_{R}-\Psi_{L}=\Delta \varphi .
$$

Analysis of (12) shows that the phase difference $\Delta \Psi_{R L}$ between orthogonal polarized components $\dot{E}_{L}$ and $\dot{E}_{R}$ is equal to the sought-for phase difference $\Delta \varphi$ between the orthogonal polarized radio beacon signals at the point of the reception - at the input of the MO receiving antenna.

The MO bearing angle $\alpha$ is calculated by putting (12) in (1):

$$
\alpha= \pm \arcsin \left[\frac{\lambda}{2 \pi d}\left(\Delta \Psi_{R L} \pm \pi n\right)\right],
$$

where $n=0,1,2, \ldots$

\section{Conclusion}

1. In case when orthogonal polarized by circle beacon signals are received onboard a moving object within the linearly polarized basis information about the bearing angle is in amplitude relations of the sin-phase orthogonal linearly polarized components of the received resulting wave.

2. In case when orthogonal polarized by circle beacon signals are received onboard a moving object within the circled polarized basis information about the bearing angle is in phase relations of the sinphase orthogonal linearly polarized components of the received resulting wave.

3. Choice of the polarization basis in which should be presented the illuminated by the beacon and received on board the moving object vector signals depends on the peculiarities of the technical problem under solution and by the mathematic analysis comfort.

\section{Acknowledgments}

This study was supported by the Ministry of Science and Education of the Russian Federation, project no. FEWM-2020-0039.

\section{References}

[1] Skrypnik O N 2019 Radio Navigation Systems for Airports and Airways (Springer) p 239

[2] Kozlov A I, Logvyn A I and Sarychev V A 2007 Radiowave Polarization "Radar Polarimetry" (Moscow, Radiotehnika) p 638 (in Russian) 
[3] Gulko V 2012 Radionavigation system for measuring the bearing of a moving object Patent $R U 2507530$

[4] Gulko V and Mescheryakov A 2017. The use of orthogonal circularly polarized beacon signals to determine the bearing of the mobile unit board UHF dual-channel receiver system. Proceedings of the XXIII International Scientific and Technical Conference "Radiolocation, navigation, communications" Voronezh p 822-826

[5] Azzam R M A and Bashara N M 1977 Ellipsometry and Polarized Light (North-Holland, Amsterdam) p 529 